\title{
Sustitución de la sabana nativa con plantaciones de Pinus caribaea (Pinaceae) en Venezuela: efecto sobre parámetros indicadores de cambios en el carbono del suelo
}

\author{
Yrma Gómez ${ }^{1}$, Jorge Paolini² \& Rosa Mary Hernández ${ }^{3}$ \\ 1. Universidad de Oriente, Laboratorio de Investigaciones Biológicas, Apdo. 1231, Puerto La Cruz, Estado Anzoátegui, \\ Venezuela; irmagomez52@hotmail.com \\ 2. Instituto Venezolano de Investigaciones Científicas Centro de Ecología, Apdo. 21827, Caracas 1020-A, Venezuela; \\ jpaolini@ivic.ve \\ 3. Universidad Simón Rodríguez IDECYT, Laboratorio de Biogeoquímica, Apdo. 47925, Caracas 1041-A, Venezuela; \\ rhernandez@reaccium.ve
}

Recibido 17-I-2008. Corregido 30-VI-2008. Aceptado 31-VII-2008.

\begin{abstract}
Substitution of native savanna by Pinus caribaea (Pinaceae) plantations in Venezuela: effect on parameters that indicated changes in soil carbon content. A great extension (615 000 ha) of native savannas of the eastern plains of Venezuela have been replaced by plantations of Pinus caribaea var. hondurensis; however, only scarce information exists about the impact of this land use on carbon dynamics in soils. We studied the effect of temporal variability and the substitution on the total organic carbon (TOC), water-soluble carbon (WSC), microbial biomass $\mathrm{C}$ (Cmic), basal respiration (BR), microbial metabolic quotient $\left(\mathrm{qCO}_{2}\right)$ and $\mathrm{Cmic} / \mathrm{Corg}$ ratio. Selected chemical properties and biological parameters of soils were measured under 3 and 29 year old forest plantations of $P$. caribaea and an adjacent native savanna which was considered the control. At each site, nine soil composite samples were collected $(0-10 \mathrm{~cm}$ depth). The studied parameters did not show a defined pattern in relation to temporal variability. Higher carbon preservation occurs in soil microbial biomass under pine plantations. The basal respiration rate and $\mathrm{qCO}_{2}$ suggests that there is a more efficient microbial carbon utilization in the plantations. The Cmic/Corg ratio shows that the microbial biomass has an important stock of soil carbon in pine plantations. The conversion of savannas to pine plantations can increase soil carbon in the eastern plains of Venezuela, but this must be balanced with the ecological importance of natural savannas ecosystems. Rev. Biol. Trop. 56 (4): 2041-2053. Epub 2008 December 12.
\end{abstract}

Key words: Carbon, Pinus caribaea, savanna, soil quality indicators, temporal variability.

La región de los llanos ocupa la faja central de Venezuela, cubre un área de 260000 $\mathrm{km}^{2}$ y representa el $35 \%$ del área total del país (Cárdenas et al. 2000). La vegetación de las sabanas orientales de Venezuela es típicamente herbácea, dominada por especies de Trachypogon y algunas arbustivas (Curatella americana, Byrsonima crassifolia) (Ramia 1967, San José y Montes 1989).

Estas sabanas poseen suelos de texturas predominantemente arenosas, fuerte acidez y bajos contenidos de materia orgánica y nutrientes, características que le imponen severas limitaciones por fertilidad (García y Herrera 1978, Rodríguez et al. 1996). Éstas han sido incluidas dentro del grupo de sabanas de baja bioproductividad (1. $\left.000 \mathrm{~g} / \mathrm{m}^{2} / \mathrm{año}\right)$, producto del estrés hídrico durante la temporada seca y del estrés físico y nutricional del suelo (San José y Montes 1989). La forma tradicional de uso de estas sabanas ha estado dedicada a la ganadería extensiva, caracterizada por emplear grandes extensiones de pasto natural de bajo valor nutritivo, y baja carga animal por hectárea (Navarro 1988). Sin embargo, la creciente presión antrópica sobre estas sabanas, para 
obtener una mayor producción de carne y leche, ha estimulado la introducción de gramíneas forrajeras exóticas, principalmente especies del género Brachiaria, que a través de un manejo que incluye encalado y fertilización, han sido efectivas en el mejoramiento de la producción y productividad animal (Rodríguez et al. 1996). Este manejo del suelo trae asociado el uso obligado de los fertilizantes con el impacto económico por el alto costo de los mismos y además, con el efecto nocivo que éstos pueden provocar durante su infiltración hacia capas profundas del suelo debido su textura arenosa.

Otro cambio de uso de la tierra en estas sabanas de baja bioproductividad, ha sido el implementado desde 1971 por la Corporación Venezolana de Guayana (CVG) y el Ministerio de Agricultura y Cría (MAC), quienes introdujeron plantaciones de pino caribe (Pinus caribaea var. hondurensis Barr. y Golf) con fines industriales. El pino caribe es autóctono de la parte continental de América Central y se emplea en planes de reforestación debido a su plasticidad ecológica, adaptabilidad a condiciones adversas, fácil manejo y crecimiento rápido.

Hasta el año 2000 las plantaciones de pino caribe en los Llanos Orientales de Venezuela abarcaban una extensión de 615000 has, lo cual representa la mayor área del trópico con una plantación forestal monoespecífica (Cedeño et al 2001). A pesar de la importancia de este proyecto forestal son pocos los estudios realizados en Uverito. En su mayoría han estado dirigidos a la caracterización de las propiedades fisicoquímicas de estos suelos, su impacto sobre la productividad (Brito et al. 1975; Fassbender et al. 1979; Marqués et al. 1994) y sobre el estudio de la descomposición de hojarascas (Bastardo et al. 1982). Es poco lo que se conoce sobre el impacto que este cambio de uso de la tierra tiene sobre el contenido de carbono orgánico en los suelos de Uverito (Campos 1999). En este sentido, resulta de gran interés determinar través de diversos parámetros microbiológicos indicadores de cambios en la calidad del suelo, cuál es el efecto de la conversión de la sabana nativa de Uverito en bosques de pino caribe sobre el carbono orgánico del suelo. Esta determinación contribuirá a la evaluación de la sostenibilidad de las plantaciones de pino en las sabanas orientales de Venezuela.

\section{MATERIALES Y MÉTODOS}

Área de estudio: El área de estudio está localizada en Uverito, Estado Monagas, Venezuela a una altitud entre 50-60 m por encima del nivel del mar. Ésta corresponde a una antigua altiplanicie del Pleistoceno conocida como Mesa de Guanipa, la cual está caracterizada por suelos altamente meteorizados y de baja fertilidad (Brito et al. 1975). El contenido de $\mathrm{C}$ y $\mathrm{N}$ total en estos suelos no sobrepasa el $10 \mathrm{~g} \mathrm{~kg}^{-1}$ y el $0.8 \mathrm{~g} \mathrm{~kg}^{-1}$, respectivamente y la capacidad de intercambio catiónico es limitante $(3 \mathrm{meq} / 100 \mathrm{~g})$. Son suelos profundos y franco-arenosos con una capacidad de campo de $50 \mathrm{~mm}$ (Pomenta 1981) y un drenaje interno generalmente excesivo. El suelo es ácido ( $\mathrm{pH} 4.2$ - 4. 8) y se caracteriza por poseer un horizonte argílico (Aa, FAa) a una profundidad que varía entre 40 y $150 \mathrm{~cm}$, característica usada como un criterio operacional de clasificación (Fassbender et al. 1979). Los suelos han sido clasificados como Arenic Haplustox, Psamentic Haplustox and Oxic Haplustults (Márquez et al. 1994). El Cuadro1 resume las características físicas y químicas predominantes de los suelos Uverito.

El clima es de bosque seco tropical. El área recibe un promedio de lluvia anual de $1134 \mathrm{~mm}$ (Visáez 2004). La temporada lluviosa es de alta intensidad y corta duración y ocurre entre Mayo y Diciembre (Brito et al. 1975; Márquez et al. 1994. ) El período seco incluye Enero a Abril. El promedio total de evapotranspiración para el período estudiado fue de 1931 $\mathrm{mm}$. La media anual de la temperatura del aire varió entre 22.4 a $32.7{ }^{\circ} \mathrm{C}$, y la temperatura del suelo registrada en los primeros $10 \mathrm{~cm}$ de profundidad varió de 34.5 a $28.1{ }^{\circ} \mathrm{C}$ durante los períodos secos y lluviosos respectivamente (Visáez 2004).

Historia de la plantación: Durante el período inicial del establecimiento de las 
plantaciones de pino caribe en Uverito, la sabana nativa no era sometida a la acción del fuego y no se hacía ninguna preparación previa del terreno. Las operaciones de siembra consistían en la abertura de surcos y distribución de las plantas en una operación combinada. Éstas eran sembradas directamente en el suelo en envases de fieltro asfáltico (Lawrence y Woods 1979, comunicación personal Ing. Forestal Rámón Díaz, PROFORCA 2001), posteriormente, este sistema de siembra fue modificado para el establecimiento de futuras plantaciones, para lo cual las parcelas se sometían a la acción de un fuego "controlado" y a una labranza doble (hasta $25 \mathrm{~cm}$ de profundidad) y se procedía a la siembra en forma mecanizada a raíz "desnuda". En la actualidad las plantas son sembradas en forma manual o mecanizada a raíz cubierta, lo cual consiste en el desarrollo del sistema radicular de las plantas en un sustrato orgánico inerte, dispuesto dentro de un contenedor plástico (tubetes). Durante la etapa de crecimiento se realiza fertilización mediante riego automático por aspersión (Anónimo 2004). Las plántulas son inoculadas con el hongo Pisolithus tinctorius.

Para el momento del muestreo, la plantación de 3 años de edad, presentaba signos de invasión por la gramínea Rynchelytrum repens, cuya presencia ha sido favorecida por el proceso de eliminación de la cobertura nativa, la cual suele emplearse antes de la siembra de las plántulas de pino. La parcela de 29 años de edad, además de presentar el estrato de acículas característico de los pinares de esta edad, estaba invadida por diversas especies vegetales no nativas y de poca incidencia, lo cual está asociado al efecto del "raleo" o "aclareo", actividad que permite remover aquellos individuos suprimidos o comercialmente no aceptables (Torres et al. 1994).

En el área de estudio administrada por la empresa Corporación Venezolana de GuayanaProductos Forestales de Oriente (CVGPROFORCA) se seleccionaron al azar tres parcelas de $100 \times 20 \mathrm{~m}^{2}$ Una de ellas representada por la sabana nativa $\left(8^{\circ} 31^{\prime} \mathrm{N}, 62^{\circ}\right.$ $\left.38^{\prime} \mathrm{W}\right)$ considerada como control, las otras correspondieron a plantaciones de $P$. caribaea de 3 años $\left(8^{\circ} 37^{\prime} \mathrm{N}, 62^{\circ} 38^{\prime} \mathrm{W}\right)$ y 29 años $\left(8^{\circ}\right.$ $\left.39^{\prime} \mathrm{N}, 62^{\circ} 38^{\prime} \mathrm{W}\right)$. En cada una de ellas se demarcaron 3 transeptos, a lo largo de los cuales se establecieron 5 puntos de muestreo. En cada punto de muestreo se tomaron muestras por triplicado. Las muestras se homogenizaron para formar tres muestras compuestas por transepto, para un total de 9 muestras compuestas por parcela. Las muestras de suelo fueron recolectadas con un barreno a una profundidad de 0-10 cm. La hojarasca del piso del bosque fue removida previo al muestreo. Las muestras fueron colocadas en bolsas plásticas, transferidas al laboratorio, tamizadas a $2 \mathrm{~mm}$ y almacenadas a $4{ }^{\circ} \mathrm{C}$ hasta su análisis.

Análisis físico y químico del suelo: $\mathrm{El}$ contenido de humedad se determinó mediante un analizador de humedad y el $\mathrm{pH}$ del suelo se estimó en agua (1:5). La determinación del carbono orgánico total se llevó a cabo por el método de la oxidación del dicromato de Walkey-Black (Anderson e Ingram 1993), el N total, por el método de Kjeldal (Bremner 1965) y la concentración de $\mathrm{P}$ total, por el método colorimétrico del azul de molibdeno (Murphy y Riley 1962).

Biomasa microbiana: la biomasa microbiana es un componente sensible y dinámico a cambios en la calidad del suelo (Haynes y Tregurtha 1999, Sánchez y Valero 2002), El carbono microbiano (Cmic) se determinó por el método de la fumigación-extracción (Sparling y West 1988). Se estabilizaron por 5 días triplicados de 20 gramos de suelo a humedad de campo, posteriormente se fumigaron con cloroformo libre de alcohol (Jenkinson y Polwson 1976) e se incubaron por $24 \mathrm{~h}$; los controles permanecieron sin fumigar. Una vez evacuado el cloroformo, las muestras se sometieron a extracción con $100 \mathrm{ml}$ de $\mathrm{K}_{2} \mathrm{SO}_{4}(0.5 \mathrm{M})$.

El Cmic fue calculado por diferencia entre el C extraído de la solución del suelo de las muestras fumigadas y no fumigadas. Para el cálculo del carbono microbiano se usó el factor de conversión propuesto por Vance et al. (1987). 
La respiración basal: la respiración basal (RB) es una medida de la actividad microbiana, de la tasa de descomposición de la materia orgánica y de los cambios en la calidad del suelo (Anderson 1982; Brookes et al. 1985; Palma et al. 2000). Ésta se determinó colorimétricamente (Alef y Nannipieri 1995). Para ello, las muestras de suelo a humedad de campo fueron cernidas y estabilizadas a temperatura ambiente por 5 días. Se colocaron $50 \mathrm{~g}$ de suelo en recipientes de $500 \mathrm{ml}$ de capacidad, se sellaron herméticamente y se incubaron por $24 \mathrm{~h}$. El $\mathrm{CO}_{2}$ liberado se atrapó en una solución de $\mathrm{NaOH}(0.1 \mathrm{~N})$, y el $\mathrm{CO}_{2}$ absorbido se tituló con HCL $(0.1 \mathrm{~N})$. Se emplearon recipientes vacíos con trampas de álcali para determinar el contenido del $\mathrm{CO}_{2}$ del aire. Los resultados se expresaron en mg C-CO $\mathrm{kg}^{-1} 24 \mathrm{~h}^{-1}$.

El cociente metabólico $\left(\mathrm{qCO}_{2}\right)$ : Es un parámetro indirecto el cual indica la eficiencia de la biomasa microbiana en la utilización del carbono del suelo (Palma et al. 2000). Se determinó mediante la relación entre el $\mathrm{CO}_{2}$ emitido durante la respiración basal y el C de la biomasa microbiana (Anderson y Domsch 1985).

La relación Cmic/Corg: Al igual que el $\mathrm{qCO}_{2}$ es un parámetro indirecto que constituye una medida satisfactoria para monitorizar cambios de la materia orgánica en el suelo (García et al. 2002, Oberson et al. 2001).

Los datos se trataron estadísticamente mediante un análisis de ANOVA de dos vías para determinar las diferencias entre las medias provocadas por efecto de la variabilidad temporal, el cambio de uso de la tierra y el efecto interactivo de ambos factores. Un análisis de correlación múltiple se empleó para determinar la relación entre los diferentes parámetros estudiados. Los análisis estadísticos se realizaron a través del programa estadístico SPSS para Windows.

\section{RESULTADOS}

Carbono orgánico total e hidrosoluble: En la sabana nativa de Uverito el COT y CHS no mostraron variación temporal significativa; mientras que en el sistema de pinares, aunque se observó una tendencia a la disminución del COT durante el período lluvioso, éste sólo disminuyó significativamente $(\mathrm{F}=9.10, \mathrm{p}<0.05)$ en el pinar de 29 años (2.84 a $2.19 \mathrm{~g} \mathrm{~kg}^{-1}$ ) (Fig. 1 A). Un comportamiento similar experimentó el CHS, el cual sólo disminuyó significativamente en la plantación de 3 años $(\mathrm{F}=0.26$, $\mathrm{p}<0.01$ ) durante este período (Fig. $1 \mathrm{~B}$ ). La sustitución de la sabana nativa por el sistema de pinares afectó significativamente $(\mathrm{F}=13.86$, $\mathrm{p}<0$. 01) el contenido de COT y de CHS en el suelos, éstos fueron mayores en el sistema de pinares de 29 años (Fig. 1 A y B).

Biomasa microbiana: La variación temporal no afectó significativamente el contenido de carbono microbiano en el suelo de la sabana nativa y de las plantaciones de pino caribe en Uverito (Fig. 2). En contraste, el cambio de uso de la tierra, es decir la conversión de sabanas a pinares y la edad de la plantación, afectaron en forma altamente significativa $(\mathrm{F}=22.27$, $\mathrm{p}<0$. 001) el contenido del Cmic en el suelo, el cual presentó los valores mas bajos en la sabana nativa (64. 1 - 68. $2 \mathrm{mg} \mathrm{kg}^{-1}$ ). El Cmic de la sabana nativa difirió significativamente de aquel presente en las plantaciones de pinos de $3(p<0.05)$ y 29 años de edad $(p<0.001)$. Éste último presentó el mayor contenido del mismo (250. 7 - 272. $9 \mathrm{mg} \mathrm{kg}^{-1}$ ) (Fig. 2).

Respiración basal: La variabilidad temporal afectó significativamente $(\mathrm{F}=32.71$, $\mathrm{p}<0$. 001) la tasa de respiración de las poblaciones microbianas presentes en el suelo de la sabana nativa y las plantaciones de pinos estudiadas en Uverito (Fig. 3 A). En todos los casos, esta actividad fue mayor durante el período de lluvias. Estos valores variaron de 5. 9 a 9.0 mg C-CO $\mathrm{kg}^{-1} 24 \mathrm{~h}^{-1}$, en la sabana nativa; de 5.6 a $12.0 \mathrm{mg} \mathrm{C}-\mathrm{CO}_{2} \mathrm{~kg}_{-}{ }^{1} 24 \mathrm{~h}^{-1}$, en la plantación de 3 años de edad; y de 9.0 a 13. $4 \mathrm{mg} \mathrm{C}-\mathrm{CO}_{2} \mathrm{~kg}^{-1} 24 \mathrm{~h}^{-1}$, en la plantación de 29 años de edad.

Cociente metabólico: Se ha señalado que la variación temporal del $\mathrm{qCO}_{2}$ es mayor que la 

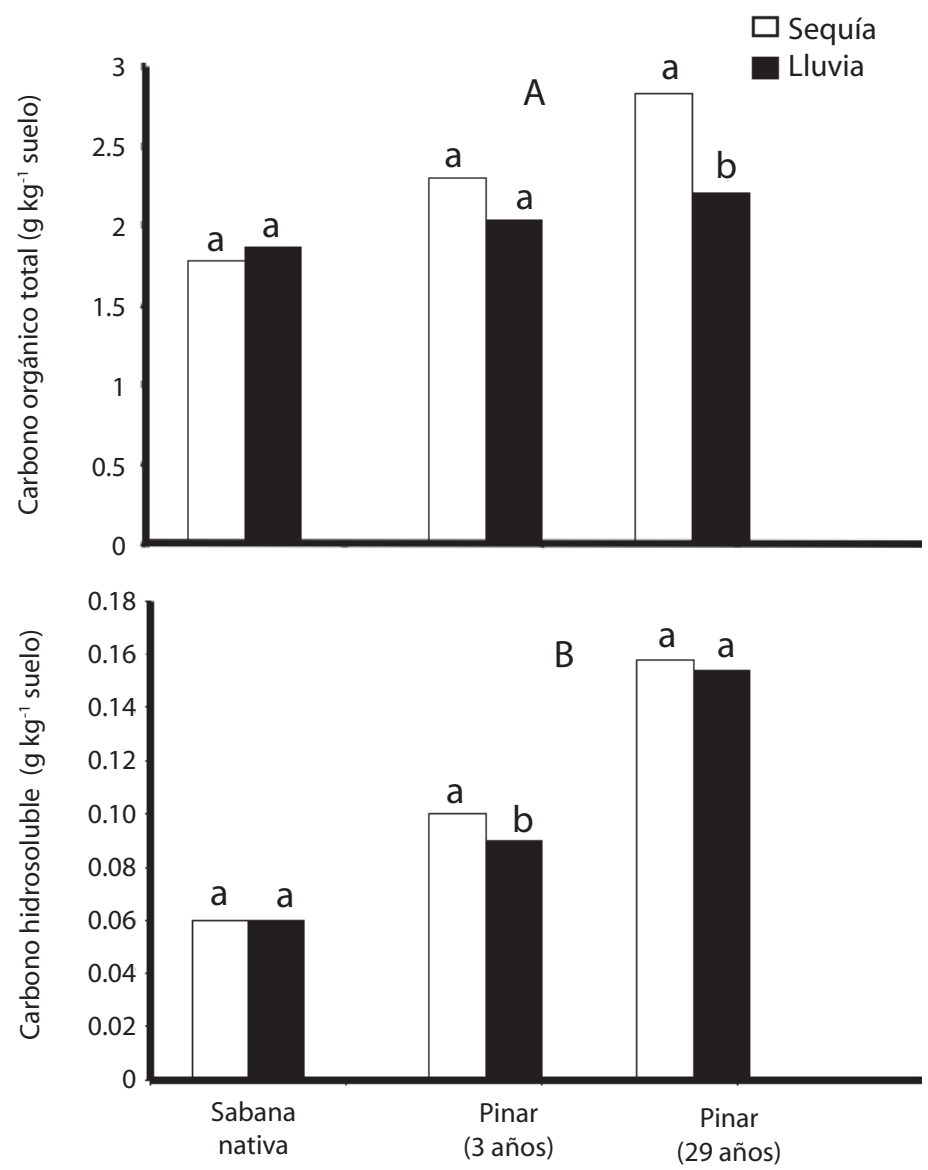

Fig. 1. (A.) Efecto de la variabilidad temporal sobre el carbono orgánico total y (B. ) el carbono hidrosoluble presentes en la sabana nativa y las plantaciones de pino de los llanos orientales Venezuela. La media entre períodos climáticos para el mismo sistema vegetal seguidos por diferentes letras minúsculas son significativamente diferentes $\mathrm{p}<0$. 05 .

Fig. 1. (A) Effect of the temporary variability on the total organic carbon and (B) water-soluble carbon present in the native savanna and pine plantations from the eastern plains in Venezuela. The averages between climate periods for the same plant system followed by different lower case letters are significantly different $(p<0.05)$.

del Cmic (Santuckova 1992). Sin embargo, en esta investigación no se observó una variación significativa de este parámetro con la temporada climática; aunque mostró una tendencia a disminuir durante la temporada lluviosa, en las plantaciones de 3 años $(0.15$ a $0.087 \mathrm{mg}$ $\mathrm{C}-\mathrm{CO}_{2}(\mathrm{mg} \mathrm{Cmic} 24 \mathrm{~h})^{-1}$ y 29 años $(0.07$ a 0. $05 \mathrm{mg} \mathrm{C}-\mathrm{CO}_{2}(\mathrm{mg} \mathrm{Cmic} 24 \mathrm{~h})^{-1}$ (Fig. $\left.3 \mathrm{~B}\right)$.

$\mathrm{Al}$ comparar el valor del $\mathrm{qCO}_{2}$ registrado en el suelo de la sabana nativa con aquel presente en los sistemas de pinares, éste sólo se diferenció $(\mathrm{p}<0.001)$ del obtenido en la plantación de pinos de 29 años (Fig. 3 B).

Cmic/Corg: La temporada climática no ejerció un efecto significativo sobre la relación Cmic/Corg del suelo de la sabana nativa. En contraste, en las plantaciones de pinos de 3 y 29 años, esta relación varió en forma significativa $(\mathrm{F}=2.68, \mathrm{p}<0.05$ y $\mathrm{F}=0.723, \mathrm{p}<0$. 001 , respectivamente) durante las temporadas de sequía y lluvia (Fig. 4). En la plantación de 


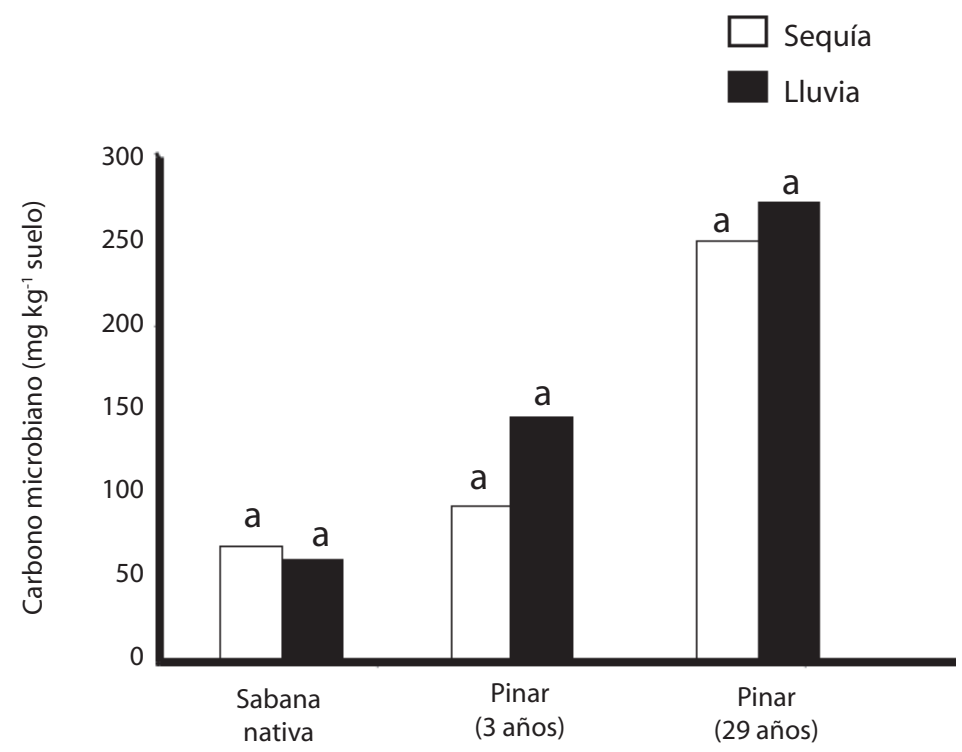

Fig. 2. Efecto de la variabilidad temporal sobre el carbono microbiano presente en la sabana nativa y las plantaciones de pino de los llanos orientales Venezuela. La media entre períodos climáticos para el mismo sistema vegetal seguidos por diferentes letras minúsculas son significativamente diferentes $\mathrm{p}<0.05$.

Fig. 2. Effect of the temporary variability on the microbian carbon present in the native savanna and pine plantations from the eastern plains in Venezuela. The averages between climate periods for the same plant system followed by different lower case letters are significantly different $(p<0.05)$.

pinos de 3 años la variación fue de 4.1 a $7.6 \%$, respectivamente, y en la plantación de 29 años de 8.3 a $12.6 \%$, respectivamente.

La sustitución de la sabana nativa por las plantaciones de pino caribe, en Uverito, afectó significativamente $(\mathrm{p}<0.001)$ la relación $\mathrm{Cmic} /$ Corg, la cual fue mayor en las plantaciones de pinos (Fig. 4).

\section{DISCUSIÓN}

Al igual que el COT, el contenido del CHS presente en los suelos de la sabana nativa y de las plantaciones de pinos en Uverito, resultó extremadamente bajo (0.06 y $0.16 \mathrm{~g} \mathrm{~kg}^{-1}$, respectivamente) (Fig. $1 \mathrm{~B}$ ), lo cual sugiere, que su presencia en estos suelos es un factor altamente limitante para la actividad microbiana. En general, la disminución del carbono en las plantaciones de pinos durante la temporada húmeda está relacionada en parte, a las mejores condiciones de humedad y temperatura que se presentan en estos sistemas, las cuales favorecen una mayor mineralización de la materia orgánica no descompuesta que permanece acumulada durante el período de sequía (Bottner 1985).

El efecto significativo de la sustitución de la sabana nativa por plantaciones de pinos sobre el contenido de carbono en el suelo, podría explicarse en el caso de la plantación de 3 años a la contribución que hace la masa de raíces finas de las plantas jóvenes de pino, las cuales pueden contribuir con un 30 al $40 \%$ del pool del carbono orgánico del suelo (He et al. 1997). Asociado a ello, está el crecimiento de la gramínea Rynchelytrum repens que invade esta parcela, lo cual contribuye con su biomasa vegetal subterránea a la preservación de una parte del carbono presente en el suelo de esta plantación. 

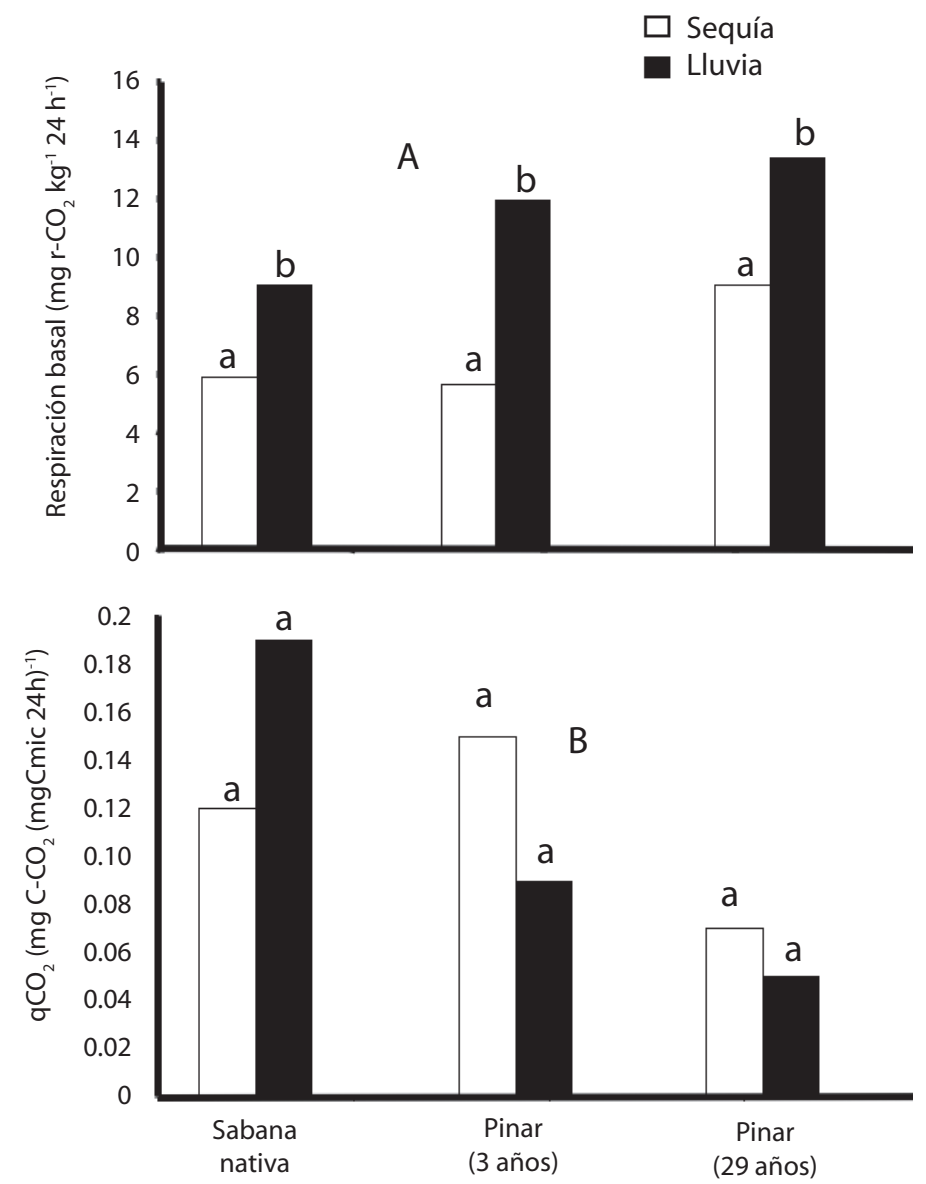

Fig. 3. (A.) Efecto de la variabilidad temporal sobre la respiración basal y (B. ) el cociente metabólico presentes en la sabana nativa y las plantaciones de pino de los llanos orientales Venezuela. La media entre períodos climáticos para el mismo sistema vegetal seguidos por diferentes letras minúsculas son significativamente diferentes $\mathrm{p}<0.05$.

Fig. 3. (A) Effect of the temporary variability on the basal respiration and (B) the metabolic quotient present in the native savanna and pine plantations from the eastern plains in Venezuela. The averages between climate periods for the same plant system followed by different lower case letters are significantly different $(p<0.05)$.

El mayor contenido de COT y CHS presente en la plantación de 29 años de edad con respecto a la sabana nativa, (Figs. 1A y B), confirma los señalamientos de Mendham et al. (2002) sobre estudios realizados en pinares desarrollados en Australia. Este incremento de la concentración de COT y el CHS en este rodal, está asociado al mayor contenido de acículas acumuladas en la superficie del suelo mineral, al reducido ciclaje y al largo período de residencia de la materia orgánica en estas plantaciones (West et al. 1988). Aunque, un aporte adicional puede ser atribuible al estrato herbáceo y de otras especies vegetales, de menor densidad, presentes en esta parcela por efecto de la actividad del "raleo" o "aclareo"; actividad que permite la entrada de luz hacia el suelo favoreciendo el desarrollo de esta vegetación, la cual contribuye con la proliferación de raíces finas, a la estabilidad de los agregados y a la preservación del carbono en el suelo (John et al. 2002). 


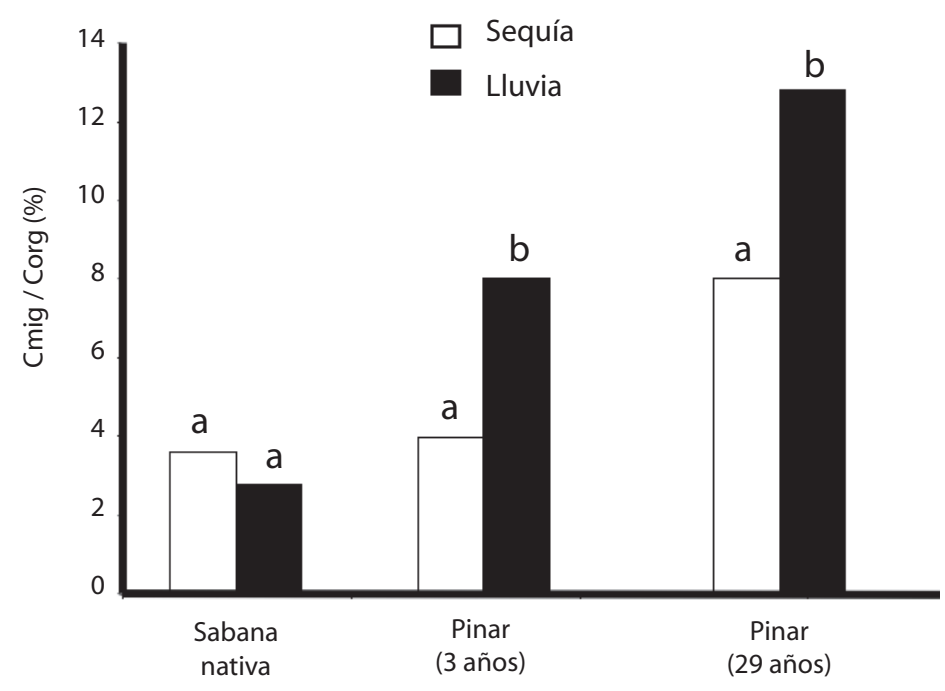

Fig. 4. Efecto de la variabilidad temporal sobre la relación Cmic/Corg presente en la sabana nativa y las plantaciones de pino de los llanos orientales Venezuela. La media entre períodos climáticos para el mismo sistema vegetal seguidos por diferentes letras minúsculas son significativamente diferentes $\mathrm{p}<0.05$

Fig. 4. Effect of the temporary variability on the Cmic/Corg relationship present in the native savanna and pine plantations from the eastern plains in Venezuela. The averages between climate periods for the same plant system followed by different lower case letters are significantly different $(p<0.05)$.

Biomasa microbiana: La ausencia de una variación temporal significativa de la biomasa microbiana en los sistemas estudiados se corresponde con los informes de Luizao et al. (1992) y Hirai et al. (2002) en estudios realizados para suelos de zonas tropicales.

El incremento de la biomasa microbiana provocado por la sustitución de la sabana nativa por plantaciones de pino caribe en Uverito, se explica por las mejores condiciones microclimáticas y el mayor contenido de carbono orgánico total e hidrosoluble presentes en el pinar con respecto a la sabana nativa; así como a la composición y eficiencia de la comunidad microbiana en la utilización del sustrato disponible presente en este sistema.

Evidentemente, la calidad de la materia orgánica incorporada al suelo por la sabana nativa y las plantaciones de pinos, influye en gran medida sobre la biomasa microbiana y su composición en estos suelos. Probablemente, esta mayor biomasa microbiana presente en las plantaciones de pino caribe esté determinada más, por una mayor proporción de hongos activos que de bacterias, ya que estos grupos de organismos descomponen en forma más eficiente sustratos de baja calidad (ricos en lignina y con una alta relación $\mathrm{C} / \mathrm{N}$ ) y los subproductos de la descomposición característicos de estos sustratos (Paul y Clark 1989). La dominancia del componente fúngico en suelos forestales ha sido determinada por diversos estudios con inhibidores selectivos (Lin y Brookes 1999).

La mayor biomasa microbiana registrada en el sistema de pinares, sugiere que una cantidad significativa de carbono, se encuentra preservada en los microorganismos del suelo de esta zona; por lo que el sistema de pinares podría contribuir no sólo con su biomasa aérea y subterránea, sino también con la biomasa microbiana del suelo, a la captura de parte del $\mathrm{CO}_{2}$ emitido hacia la atmósfera, por la actividad mineralizadora del carbono que se produce en la sabana nativa. 
Respiración basal: Si se considera que la tasa de respiración del suelo, es una estimación de la actividad microbiana total en éste, los valores obtenidos en esta investigación confirman, que la actividad microbiana en los suelos de los llanos orientales de Venezuela es sumamente baja, característica que está determinada no sólo por el bajo contenido de carbono en los mismos; sino también por las condiciones edafo-climáticas existentes.

A pesar de la baja actividad respiratoria que se registró en los suelos estudiados, se observó un incremento significativo $(\mathrm{p}<0.001)$ de la respiración basal durante el período lluvioso (Fig. $3 \mathrm{~A}$ ), lo cual evidencia que en estos suelos arenosos, caracterizados por su bajo contenido de humedad, el ligero pero significativo incremento del mismo juega un papel muy importante en la actividad microbiana. Es posible que el inicio de las lluvias estimule el metabolismo de los sustratos orgánicos procedentes de los residuos, y de la muerte de los microorganismos ocurrida durante la sequía, lo que explica en parte la disminución del carbono durante el período lluvioso (Fig. 2A y B). Estos resultados confirman los informes de Luizao et al. (1992) sobre una mayor actividad respiratoria en períodos húmedos, y los señalamientos referentes a una mayor tasa de mineralización del carbono, durante el rehumedecimiento de los suelos (Birch 1960).

El cambio de uso de la tierra provocó un incremento de la respiración basal, que fue significativo en la plantación de 29 años (Fig. 3 A). La mayor actividad que se produjo en esta plantación, está relacionada en parte no sólo al mayor contenido de humedad del suelo (4. 1\%), que se registra en esta parcela en comparación con la sabana nativa (2. 5\%), sino también a la mayor concentración de carbono (Figs. 1 A y B) y de biomasa microbiana presente en estas plantaciones (Fig. 2).

Cociente metabólico: A pesar de que en el sistema de pinares existe un alto contenido de lignina, bajo contenido de $\mathrm{N}$ y por ende una baja calidad de la materia orgánica (Pastor et al. 1987), características que afectan la actividad biológica del suelo, el $\mathrm{qCO}_{2}$ resultó menor que aquel registrado para la sabana nativa (Fig. 3 B), lo que significa, que a pesar de la baja calidad de los residuos presentes en esta zona, existe en ella una biomasa microbiana más eficiente en la utilización del carbono para la síntesis celular (Ross et al. 1999, Saggar et al. 2001). Se ha señalado que la biomasa fúngica, la cual suele dominar en el sistema de pinares (Lin y Brookes 1999), es más eficiente que el componente bacteriano en la utilización del carbono por unidad de carbono respirada (Wardle y Ghani 1995).

Cmic/Corg: Aunque los valores de la relación $\mathrm{Cmic} /$ Corg obtenidos en esta investigación fueron relativamente altos, éstos están en el ámbito de los señalados por Saggar (2001), Haynes y Tregurtha (1999) y Turner et al. (2002). Los valores de esta relación obtenidos en las plantaciones de pinos de 3 y 29 años durante la temporada lluviosa (Fig. 4), confirman que un mayor contenido de humedad resulta en un incremento de la misma (Insam 1990).

Si se considera que la relación $\mathrm{Cmic/Corg}$ asocia la biomasa microbiana a la dinámica del carbono en el suelo y por ende su disponibilidad en el mismo (Gallardo y Schlesinger 1994), y que ésta representa la proporción de Corg contenido en la biomasa microbiana (Turner et al. 2002), los valores mayores de esta relación obtenidos en la plantación de 29 años confirman los resultados del $\mathrm{qCO}_{2}$, referentes a que las poblaciones microbianas presentes en esta plantación, realizan un uso más eficiente del carbono.

Correlaciones: El Cmic se correlacionó positiva y significativamente con el COT $(\mathrm{r}=$ $\left.0.648^{* *}\right)$ y el CHS $\left(\mathrm{r}=0.615^{* *}\right)$ del suelo. Esta estrecha correlación es comprensible, ya que tanto el carbono orgánico como la biomasa microbiana son componentes importantes del pool de la materia orgánica del suelo y confirman que la biomasa microbiana del suelo está usualmente correlacionada al contenido de la materia orgánica del mismo (Vance et al. 1987, 
Tate 2000, Turner et al. 2002). Esta correlación sugiere, que en estos suelos existe un balance entre la biomasa microbiana y el contenido de materia orgánica en los mismos.

El Cmic se correlacionó positivamente con la respiración basal $\left(\mathrm{r}=0.299^{*}\right)$, esto sustenta los señalamientos existentes para diferentes tipos de suelo y manejos agrícolas (Jöergensen et al. 1995, Franzluebbers et al. 1995, Leiros et al. 2000) y sugiere que la magnitud de la biomasa microbiana, desarrollada en los sistemas nativos e intervenidos con pinares en suelos de los llanos orientales, está acoplada con el grado de actividad metabólica que se produce en los mismos. En contraste a la correlaciones positivas descritas anteriormente, el Cmic se correlacionó negativamente con el $\mathrm{qCO}_{2}$ determinado en la sabana nativa y en el sistema de pinares $\left(\mathrm{r}=-0.622^{* *}\right)$. Este tipo de correlación es considerado un fenómeno común (Sparling y West 1988, West et al. 1988).

Es importante señalar, que los parámetros microbiológicos determinados en esta investigación resultaron satisfactorios para determinar cambios en la calidad del suelo por efecto de la variabilidad temporal y el cambio de uso de la tierra. Este cambio alternativo de uso de la tierra en Uverito puede considerarse ecológicamente sostenible, siempre y cuando se tomen en cuenta los criterios sobre la importancia ecológica de la sabana, la conservación a nivel del paisaje y de las áreas naturales.

La sostenibilidad ecológica determinada en esta investigación, a través de diversos parámetros microbiológicos, está basada en el potencial que tienen las plantaciones de pino caribe en Uverito para el secuestro del carbono en el suelo. Esta particularidad, aunado al potencial de estas plantaciones para fijar el $\mathrm{CO}_{2}$ en su biomasa aérea y subterránea, contribuye al esfuerzo mundial en la mitigación del calentamiento global. Asociado a ello, estas plantaciones también contribuyen a la reducción de la presión a la cual están sometidos los bosques naturales.

\section{RESUMEN}

Una gran extensión (615000 has) de las sabanas nativas de los Llanos Orientales de Venezuela está siendo reemplazada por plantaciones de Pinus caribaea var. hondurensis; sin embargo, hasta ahora existe escasa información sobre el impacto de este manejo del suelo sobre los parámetros indicadores de la dinámica del carbono en el suelo. El objetivo de esta investigación fue determinar el efecto de la variabilidad temporal y la sustitución sobre el contenido de carbono orgánico total (COT), carbono hidrosoluble (CHS), carbono microbiano (Cmic), respiración basal $(\mathrm{RB})$, cociente metabólico microbiano $\left(\mathrm{qCO}_{2}\right)$ y la relación $\mathrm{Cmic/Corg}$. Las propiedades químicas y parámetros biológicos seleccionados fueron determinados en plantaciones de $P$. caribaea de 3 y 29 años de edad y en una sabana nativa adyacente a estas plantaciones, la cual fue considerada como control. En cada sitio de muestreo recolectamos nueve muestras compuestas $(0-10 \mathrm{~cm}$ de profundidad). Los parámetros estudiados no mostraron un patrón definido in relación a la variabilidad temporal. Hay mayor preservación del carbono dentro de la biomasa microbiana presente en suelos de Uverito en plantaciones de pinos. La tasa de respiración basal y el cociente metabólico indican que en estos suelos existe una comunidad microbiana poco activa, la cual es más eficiente en la utilización del carbono en las plantaciones de pino. La relación $\mathrm{Cmic} / \mathrm{Corg}$ indica que en las plantaciones la biomasa microbiana constituye una importante reserva del carbono en el suelo. La sabana nativa a plantaciones de pino caribeño en estos suelos puede ser considerada un manejo efectivo para incrementar la calidad del suelo en los llanos orientales de Venezuela, siempre y cuando se tome en cuenta la importancia ecológica de las sabanas.

Palabras clave: Carbono, indicadores de calidad del suelo, Pinus caribaea, sabana, variabilidad temporal.

\section{REFERENCIAS}

Alef, K. \& P. Nannipieri. 1995. Methods in applied soil microbiology and biochemitry. Academic. London,United Kingdom.

Anderson, J. P. E. 1982. Soil respiration, p. 837-871. In A. L Miller \& D. R. Keeny. (eds) Methods of Soil Analysis, Part 2: Chemical and Microbiological properties. Society of agronomy and Soil Science Society of America, Madison, Wisconsin, USA

Anderson, J. M. \& J. S. Ingram. 1993. Tropical soil biology and fertility. A handbook of methods. CAB International, Wallingford, United Kingdom. 138 p. 
Anderson, T. H. \& K. H. Domsch. 1985. Determination of ecophysiological maintenance $\mathrm{C}$ requirements of soil microorganisms in a dormant state. Biol. Fertil. Soils 1: 81-89.

Bastardo, H. ,A. Esteves \& D. A. Flores. 1982. Desaparición de materia orgánica en un bosque cultivado de Pinus caribaea var hondurensis, en Venezuela. pp. 65-71. In: C. C Cerri, D. Athié \& D. Sodrzeieski. (eds. ). Coloquio Regional sobre Materia Orgánica do Solo. Piracicaba, Sao PauloBrasil.

Birch, H. F. 1960. Nitrification in soils after different periods of dryness. Plant Soil 12: 81-96.

Bottner, P. 1985. Response of microbial biomass to alternate moist and dry conditions with ${ }^{14} \mathrm{C}$ and ${ }^{15} \mathrm{~N}$ labeled plant material. Soil Biol. Biochem. 17: 329-337.

Bremner, J. M. 1965. Total nitrogen, p. 1149 -1178. In: A. Black. (ed) Methods of soil analisys, part 2. C. A Agronomy. Am. Soc. Agrom. Madison, Wisconsin, USA.

Brito, P. , J. Comerma \& R. Cañizales. 1975. Aptitud de las tierras de la zona de Chaguaramas, Estado Monagas, para la siembra de Pinus caribaea. Agron. Trop. 25: 295-304.

Brookes, P. C. , A. Landman, G. Pruden \& D. S Jenkinson. 1985. Chloroform fumigation and the release of soil nitrogen: a rapid direct extraction method to measure microbial biomass nitrogen in soil. Soil Biol. Biochem. 17: 837-842.

Campos, G. A. J. 1999. Efecto de la siembra de Pinus caribaea L. en las fracciones de la materia orgánica de un suelo de sabana. Uverito- Estado Monagas. Tesis de Licenciatura. Universidad Central de Venezuela, Venezuela.

Cárdenas, A. L. , C. R. Campos \& F. Escamilla. 2000. Geografía de Venezuela. Fondo Editorial de la Universidad Pedagógica Experimental Libertador y la Fundación Programa de Formación Docente. Caracas. Distrito Federal.

Cedeño, L. , C. Carrero, W. Franco \& A. Torres. 2001 Sphaeropsis sapinea asociado con quema de cogollo muerte regresiva y cáncer en troncos, ramas y raíces del pino caribe en Venezuela. Interciencia. 26:210215 .

Fassbender, H. W. , J. Comerma, P. Brito \& F. Salas. 1979. Estado nutricional de los suelos en la zona de plantaciones de Pinus caribaea en el Oriente de Venezuela. Acta Cient. Venezolana 30: 582-585.
Franzluebbers, A. J. , F. M. Hons \& D. A Zuberer. 1995. Tillage and crop effects on seasonal soil carbon and nitrogen dynamics. Soil Sci. Soc. Am. J. 59: 16181624.

Gallardo, A. , W. H. Schlesinger. 1994. Factors limiting microbial biomass in the mineral soil and forest floor of warm-temperate forest. Soil Biol. Biochem. 26: 1409-1415.

García, M. J. \& R. Herrera. 1978. Mineralogía, aluminio cambiable y cargas eléctricas en dos ultisoles de ecosistemas de sabana del Nor-Oriente Venezolano. Acta. Cient. Venezolana. 29: 20-24.

García, C. T. Hernández, A. Roldan \& A. Martin. 2002. Effect of plant cover decline on chemical and microbiological parameters under Mediterranean climate. Soil Biol. Biochem. 34: 635-642.

Haynes, R. J. , R. Tregurtha. 1999. Effects of increasing periods under intensive arable vegetable production on biological, chemical and physical indices of soil quality. Biol. Fertil. Soils. 28: 259-266.

He, Z. L. , J. Wu, A. G. O’Donnell \& J. K Syers1997. Seasonal responses in microbial biomass carbon, phosphorus and sulphur in soils under pasture. Biol. Fertil. Soils. 24: 421-428.

Hirai, K. , M. Takahashi, P. Limtong \& S. Suksawang. 2002. Biochemical dynamics under different types of tropical seasonal forest in Western Thailand. $17^{\text {th }}$ WCSS. Symposium n ${ }^{\circ} 14$. Thailand. p. 528-1- 528-7.

Insam, F. 1990. Are the soil microbial biomass and basal respiration governed by climatic regime?. Soil Biol. Biochem. 22: 525-532.

Jenkinson, D. S. \& D. S. Polwson, D. 1976. The effects of biocidal treatments on metabolism in soil - I. Fumigation with chloroform. Soil Biol. Biochem. 8: 167-177.

Jöergensen, R. G. , T. H. Anderson \& V. Wolters. 1995. Carbon and nitrogen relationships in the microbial biomass of soils beech (Fagus sylvatica L. ) forest. Biol. Fertil. Soils. 19: 141-147.

John, B. , H. N Pandey \& R. S Tripathi. 2002. Decomposition of fine roots of Pinus kesiya and turnover of organic matter, $\mathrm{N}$ and $\mathrm{P}$ of coarse and fine pine roots and herbaceous roots and rhizomes in subtropical pine forest stands of different ages. Biol. Fertil. Soils. 35: 238- 246 
Lawrence, W. V. \& F. W. Woods 1979. Relación de índices de calidad de sitio con factores topográficos y edáficos en plantaciones jóvenes de pino caribe en las sabanas orientales de Venezuela. Corporación Venezolana de Guayana y Universidad de los Andes. Venezuela. $26 \mathrm{p}$

Leiros, M. C. , C. Trasar-Cepeda, S. Seoane, \& F. GilSotres. 2000. Biochemical properties of acid soils under climax vegetation (Atlantic Oakwood) in an area of the European temperate-humid zone (Galicia, NW Spain): general parameters. Soil Biol. Biochem. 32: 733-745.

Lin, Q. \& P. C. Brookes. 1999. Arginine ammonification as a method to estimate soil microbial biomass and microbial community structure. Soil Biol. Biochem. 31: 1985-1997

Luizao, R. C. C. , T. A. Bonde \& T. Rosswall. 1992. Seasonal variation of soil microbial biomass- the effects of clearfelling a tropical rainforest and establishment of pasture in the central Amazon. Soil Biol. Biochem. 24: 805-813.

Márquez, O. , R. Hernández, G. W Franco, F. Visáez. 1994. Factores edáficos y estado nutricional de plantaciones de Pinus caribaea en relación a la muerte regresiva, en Uverito, Estado Monagas. Venesuelos. 2: 15-18.

Mendham, D. S. , A. M. O'Connell \& T. S Grove. 2002. Organic matter characteristics under native fores, long-term pasture, and recent conversion to Eucalyptus plantations in Wester Australia: microbial biomass, soil respiration, and permanganate oxidation. Aust. J. Soil Res. 40: 859-872.

Murphy, J. \& J. P. Riley. 1962. A modified single solution method for the determination of phosphate in natural water. Anal. Chim. Acta. 27: 31-36.

Navarro, D. L. , 1988 a. Gramíneas más usadas en el cultivo de pastos en la región Nor- Oriental FONAIAP Divulga, Caracas, Venezuela. 57 p.

Oberson, A. , D. K Friesen, I. M Rao, S. Bühler, E. Frossard. 2001. Phosphorus transformations in an Oxisol under contrasting land-use systems: The role of the soil microbial biomass. Plant Soil. 237: 197210 .

Palma, R. M. , N. M Arriego, M. J Saubidet, \& M. E. Conti. 2000. Chemical and Biochemical properties as potencial indicators of disturbance. Biol. Fertil. Soils. 32: $381-384$

Pastor, J. , R. H Gardner, V. H. Dale, \& W. M. Post. 1987. Successional changes in nitrogen availability as a potential factor contributing to spruce declines in boreal North America. Can. J. Forest Res. 17:13941400.

Paul, E. A. \& F. E Clark. 1989. Soil Microbiology and biochemistry. Academic. San Diego, California.

Pomenta, M. L. E. 1981. Efectos de los pinares de Uverito (Edo. Monagas) sobre el grado de acidificación y las formas solubles de Al, Fe y $\mathrm{Ca}$ en el perfil del suelo. Trabajo especial de Grado, Universidad Central de Venezuela. Venezuela.

Ramia, M. 1967. Tipos de sabanas de los Llanos venezolanos. Biol. Soc. Ven. Cienc. Nat. 112: 264-282.

Rodríguez, T. , D. Sanabria \& L. Navarro. 1996. Nuevos enfoques en el manejo de sabanas en los Llanos Orientales Venezolanos. FONAIAP. Venezuela.

Ross, D. J. , K. R. , Tate, N. A Scott, C. W Feljham. 1999. Land use change: effects on soil carbon nitrogen and phosphorus pools and fluxes in three adjacent ecosystems. Soil Biol. Biochem. 31: 803-813.

Saggar, S. , C. B Hedley, G. J. Salt. 2001. Soil microbial biomass, metabolic quotient, and carbon and nitrogen mineralisation in 25-years-old pinus radiate agroforestry regimes. Aust. J. Soil Res. 39: 491-504.

Sánchez, D. J. \& J. A. P. Valero. 2002. Estudio comparativo de la actividad microbiana en los suelos forestales de la sierra de Crevillente. Trabajo de Investigación Marta Goberna Estellés. Valencia, España.

San José, J. J. \& R. Montes. 1989. An assessment of regional productivity: The Trachypogon savannas at the Orinoco Llanos. Nature Res. 25: 15-18.

Santuckova, H. 1992. Microbial biomass, activity and soil respiration in relation to secondary succession. Pedobiologia. 36: 341-350.

Sparling, G. P. , A. W. West. 1988. Modifications to the fumigation-extraction technique to permit simultaneous extraction and estimation of soil microbial $\mathrm{C}$ and N. Commun. Soil Sci. Plant Anal. 19: 327-344.

Tate II, R. L. 2000. Soil Microbiology. Wiley, New York, USA.

Torres, A. \& W. Franco. 1994. Efecto de la fertilización con roca fosfórica y borax en el crecimiento de plantaciones de pinus caribaea var. hondurensis en el oriente de venezuela. Interciencia. 19:374-379.

Turner, B. L. , D. W Hopkins, P. M Haygarth, N. Ostle. 2002. $\beta$-Glucosidase activity in pasture soils. Appl. Soil Ecol. 20: 157-162. 
Vance, E. D., D. C Brookes, D. S. Jenkinson. 1987. An extraction method for measuring soil with microbial biomass C. Soil Biol. Biochem. 19: 703-707.

Visáez, F. J. 2004. Anuario Climatlógico para el período 1991- 2004. CVG-PROFORCA. Venezuela.

Wardle, D. A. \& A. Ghani. 1995. Why is the strength of relationship between pairs of methods for estiming soil microbial biomass often so variable?. Soil Biol. Biochem. 27: 821-828.
West, A. W. , G. P Sparling, T. W. Speir, \& J. M Wood. 1988. Comparison of microbial C, N-flush and ATP, and certain enzyme activities of different textured soils subject to gradual drying. Aust. J. Soil Res. 26: 217-229.

\section{REFERENCIA DE INTERNET}

Anónimo. 2004. Resumen público de certificación de Terranova de Venezuela. (www.rainforest-alliance. org/forestry/document/terranovafmpubsum04. pdf. Pdf; consultado 28 julio 2008). 
